Review

\title{
Short but continuous natural pain for depression treatment and beyond
}

\author{
Mingwei Huang*,1 \\ ${ }^{1}$ Department of Biomolecular Chemistry, School of Medicine and Public Health, \\ University of Wisconsin-Madison \\ Madison, WI 53706
}

\author{
*To whom correspondence should be addressed: \\ Dr. Mingwei Huang \\ 1135 Biochemistry Building \\ 420 Henry Mall \\ University of Wisconsin-Madison \\ Madison, WI 53706 \\ Telephone: (608) 265-5689 \\ E-mail: mhuang38@wisc.edu
}

Running Title: short but continuous pain and depression

Keywords: pain, depression, treatment, meditation, synaptic plasticity and homeostasis, DNA damage response

\section{Frequently used abbreviations:}

Major Depressive Disorder (MDD), Short but Continuous Pain (SCP), Hypothalamic-Pituitary-adrenal (HPA), Double Strand Break (DSB), DNA Damage Responses (DDR), Deep Brain Stimulation (DBS). 
Review

\begin{abstract}
The correlation and comorbidity between depression and chronic pain have been observed for a long time. Generally, it is considered that the two conditions reinforce each other, whereas the causal relationship between them is not clear. However, some evidence suggested that chronic pain may reverse the progression of depression in some cases. This article presents a selective review of clinical and pharmacological observations between depression and pain, and their interactions at neurochemical and neurobiological levels. In addition, we open a discussion on a recent case report of repeated success of using short but continuous pain during meditation as the only treatment of depression, compared to initial success but no remission with other conventional antidepressants on the same patient. Together this review proposes an updated model for depression and its various treatments that is based on synaptic and system homeostasis. More importantly, it suggests that short but continuous pain may benefit depression recovery through its properties that are different from either acute or chronic pain and represents a novel research area that has been largely neglected to date.
\end{abstract}


Review

Major depressive disorder (MDD) is a common psychiatric disorder, which leads to usually seriously impaired condition in the patients and great global disability burden $(1,2)$. Current treatments for depression often take weeks or months to demonstrate full effect, not all patients have a good response, relapse rate is high even for those effective treatment, and only a subset of patients achieved remission, raising the question of either the complexity of the disease or the effectiveness of the currently available treatments $(3,4)$.

In light of its diverse etiologies, comorbidity with many other diseases, and complex underlying pathology, it is challenging to understand the physiological basis of MDD, which may be a complex of related diseases, rather than a single one. In addition to the incomplete understanding of MDD, the individual heterogeneities among patients may render it an elusive goal to develop a universal treatment for MDD. But studying the effectiveness of each current treatment can help stratify patients into different subtypes for customized treatments as un ultimate goal (5).

Recently, we published the first report of repeated success of using meditation as the only treatment of MDD, whereas other conventional antidepressants achieved initial success but no remission on the same patient. We hypothesized

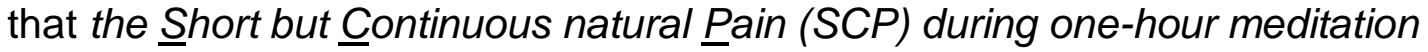
sittings has the therapeutic effect to treat depression in the case of this patient and potentially others with MDD. The special opportunity to eliminate individual heterogeneity has enabled us to probe deeply into the potential mechanisms of depression treatments and the intricate physiology of depression itself, providing profound implications in the treatment of other MDD patients. More importantly, the case report helps us to dissect one specific component of meditation for its long-known and well-established benefits against depression $(6,7)$.

However, our proposed hypothesis is counterintuitive to the current understanding of the relationship between depression and chronic pain. Chronic pain is generally detrimental, whereas acute pain is usually useful to trigger reflex to prevent injury. SCP may exhibit different properties from those two types of pain and represent an exciting novel research area that has been largely neglected to date. In this review we will present the links between depression and pain and discuss potential mechanisms of our hypothesis. Due to space constraints, we have not attempted a comprehensive review but focused on selected areas that are closely related to the interface between depression and pain. We will start with some clinical and pharmacological observations, which suggest some fundamental connections between these two conditions.

\section{Depression and chronic pain}

Depression and chronic pain are usually closely associated, but their causal relationship is not clear $(8,9)$. However, it was noted that a substantial minority of treatment seekers for chronic pain do not seem depressed, and even lower rates 
Review

of depression in community samples of people with persistent pain, where the modest relationship is proposed to be mediated by cognitive factors (catastrophizing, self-efficacy, sense of control/helplessness), physical functioning, medication variables, and psychological factors (resilience and flexibility) (10). Thus, chronic pain may not have a simply causal effect on depression, but instead might exert some antagonistic function.

In a study that prospectively examined depression response to chronic pain over a course of six years in a nationally representative middle-aged sample of 2,172 individuals, the resilience group (no or minimal depression symptoms prior to and following pain onset) was much larger than the post-pain depression group (low depression at baseline but increasing symptoms following pain onset) $(72.0 \%$ compared to $11.4 \%$ ). More strikingly, gradually declining depression symptoms were observed in a higher number of patients with initially high depression following the onset of chronic pain, compared to patients with similar depression before and after the onset of chronic pain (9.8\% vs 6.8\%) (11). These data provide some support to our hypothesis, but the complex relationship between depression and pain, and multiple involving factors demands more rigorous research.

At a physiological level, chronic pain patients show reduced levels of serotonin and dopamine and deficient working memory, and impaired decision making, which are also typical symptoms of depression(12). Even though SCP is not exactly the same as chronic pain, their shared pain pathways may act on the same targets involved in depression with different outcomes due to their different properties.

\section{Drugs in depression and pain}

\section{Conventional antidepressants}

One major difficulty with the monoamine theory of depression for conventional antidepressants is that the antidepressant effect usually takes weeks develop whereas the monoamine activity increases quickly (13). Thus additional theories, such as neurotrophic and neurogenic hypotheses, have been proposed to explain the pathophysiology of depression and corresponding antidepressant responses (5). In the case report, slowly-building antidepressant effect was not observed for multiple conventional antidepressants and/or their combinations. instead, there were two times of fast recovery through antidepressant(s) that took place within several or 36 hours after administration of the new medications. One possible explanation for this discrepancy might lie in the ability of monoamines to serve as analgesics, since descending pain pathway utilizes monoamine neurotransmitters and conventional antidepressants may actually work through enhancing the analgesic activity of that pathway (13). 
Review

In the clinics, The tricyclic antidepressants (TCAs) have been used to treat chronic pain and more specifically one TCA, amitriptyline, is commonly prescribed off label for the treatment of shingles pain (13). The serotonin/norepinephrine reuptake inhibitor (SNRI) duloxetine is approved for the treatment of fibromyalgia pain and also efficacious to treat pain in diabetic peripheral neuropathy (14). If this is the mechanism behind the recovery from depression using conventional antidepressants, the fast-acting effect of psilocybin may be also exerted through monoamine related pain pathways, since it primarily functions as an agonist of serotonin $(5-\mathrm{HT})$ receptors $(15,16)$.

\section{Ketamine}

Ketamine is a well-established anesthetic and generates "dissociative anesthesia", which is quite different from more traditional volatile-based anesthesia and includes hypnosis, intense anti-nociception, increase sympathy, and maintenance of respiration (17). Sub-anesthetic dosing of ketamine, a noncompetitive $\mathrm{N}$-methyl-D-aspartate receptor (NMDAR) antagonist, has shown rapid clinical antidepressant effect in both MDD and bipolar depression (17-27). The hypnotic effect of ketamine is largely mediated through inhibition of NMDA and $\mathrm{HCN} 1$ receptors, its sedation and analgesia effects are modulated by cholinergic, aminergic (serotonergic and noradrenergic activation and inhibition of re-uptake), and opioid systems, whereas its effects in chronic pain and depression are likely achieved through glutamate neurotransmission and secondary changes in synaptic plasticity $(17,28)$. Additionally, ketamine acts on different levels of inflammation to confer an overall anti-inflammatory effect, limiting exacerbation of systemic inflammation but not disrupting local healing processes (29), which could also be an important aspect of its antidepressant function. The responses or part of the responses of our body to the SCP could be similar to some of the physiological changes caused by ketamine and thus contribute to relief in depression symptoms.

\section{Inflammation in depression and pain}

In the case report, allergic reaction to seasonal pollens correlated with depression symptoms and the relief of allergic response marked the beginning of depression recovery triggered by SCP perception. These observations agree well with the close association of inflammation with both depression and pain and suggest possible explanations for potential causal relationships. Peripheral immune modulators could induce psychiatric symptoms in animal models and human, and in psychiatric patients, peripheral immunological abnormalities are also more prevalent compared to healthy control (30-33). It is proposed that neuroinflammation and cytokines are at least a contributing factor to the pathological development of depression (34-37).

The sympathetic nervous system (SNS) and the hypothalamic-pituitary-adrenal (HPA) axis have long been closely associated with the pathophysiology of 
Review

depression, but the causal relationship is far from clear (37). The overdrive of the HPA axis, hypersecretion of cortisol, dysfunctional feedback mechanisms, and/or cytokine over-secretion are most consistently seen in patients with more severe depression, such as melancholic or psychotic depression $(37,38)$. In the case report, SCP could serve as a sustained stressor and stimulate the HPA axis and the SNS, leading to glucocorticoid (GC) release from the adrenal glands and further anti-inflammatory effects, or acting through the parasympathetic nervous system and the cholinergic anti-inflammatory pathway (reduced production of inflammatory cytokines and relief of allergic responses) (29, 31-33). This may be similar to what happened during vagus nerve stimulation, which is a promising treatment for treatment of drug-resistant depression, most likely due to its ability to increase the vagal tone and to inhibit cytokine production $(41,42)$.

\section{Pain activation and long-lasting effect}

The multiple dimensions of pain experience (sensory, emotional, and cognitive) involve activation of different regions of brain (pain matrix), which are also involved in other functions of the brain and thus likely affected by depression. The brain areas that constitute the brain network for acute pain, most consistently revealed through functional imaging studies, are primary and secondary somatosensory cortices (S1 and S2), insular cortex (IC), anterior cingulate cortex (ACC), prefrontal cortex (PFC), and thalamus (Th), out of which chronic clinical pain conditions more frequently involve PFC and often associated with decreased baseline activity or decreased stimulus related activity in Th (4345). Also abnormalities of the white matter structures connecting the medial PFC and the nucleus accumbens were observed in chronic pain patients (46).

With a significant overlap with pain matrix, structural and functional abnormalities in PFC, ACC, hippocampus, basal ganglia, and amygdala have been documented in MDD $(47,48)$. However, meditation, where SCP was first implicated in our case report, can to certain degree reverse these abnormalities, as well as other neurochemical, neuroendocrinal, neurobiological, and immune/inflammatory changes associated with depression $(6,7)$. Last but not least, Meditation associated pain has also been described by others (49). Thus, it is reasonable to postulate that SCP may activate certain pathways or brain areas, and lead to depression relief through a mechanism that has not be revealed yet.

\section{Possible system mechanisms for the antidepressant effects of SCP}

As illustrated above, pain and depression are inter-connected at many different levels through the endocrine, immune, and nervous systems. The same systems communicate with each other to achieve a dynamic equilibrium and system homeostasis, which are exemplified at different levels, such as Th1/2 balance 
Review

and synaptic plasticity $(40,50)$. However, dysregulations triggered by stress result in shifts in balance and the system may not be able to return to homeostasis and exemplified again at different levels, such as Th1/2 imbalance and synaptic malfunctions (30). In this vein, depression is not a simple disease caused by one or several factors, but a disrupted system homeostasis. Multiple factors that influence the function of the immune and nervous systems, such as monoamines and neural synapses, can be the triggering factors that initiate the development of depression (5, 30, 36, 37, 51). Eventually, the system reaches maladaptive states that have symptomatic presentations. This could help explain the increased sensitivity to pollen during the depression and remarkably decreased allergic response as the start of recovery from depression observed in the case report.

Interestingly, in a recent transcriptomic profiling of molecular brain-based phenotypes across major psychiatric disorders, including autism, schizophrenia, bipolar disorder, depression, and alcoholism, shared molecular neuropathology (demonstrated in transcriptional dysregulation) parallels polygenic (singlenucleotide polymorphism-based) overlap, suggesting a substantial genetic causal component and pathways of molecular convergence, despite the distinct features and specificity of each condition (33). Using the analogy of a rugged funnel-like energy landscape proposed in protein folding (52), the ideal stable state of homeostasis is in the center and other less stable maladaptive states can be widely spreading across an infinite field, where the depth can also vary from person to person and change at different stages of life. Depression and other psychiatric or somatic illnesses are all individual maladaptive states and can potentially be cured by disrupting the system in a controlled way and subsequent guiding towards the center, similar to protein refolding.

Each body system is under constant stimulations of thousands of environmental and internal changes and reacting to each stimulation is neither practical nor beneficial. Thus, a balance of inhibition and perception needs to be maintained and the presentation of the balance is homeostasis at both system and synapse levels. In this vain, conventional antidepressants and psilocybin exert their fast antidepressant effect through their ability to manipulate body's response to external and internal stimuli. Similarly, ketamine as well as other new fast acting antidepressants might function through similar mechanisms. However, shifted homeostatic states of the body make it an elusive goal to achieve remission.

Possibly, during the observed repeated recovery from depression using meditation in the case report, SCP broke down the barrier set up by depression with force and restored the homeostasis of neuronal synapses and networks, demonstrating plasticity as one of their most fundamental and intrinsic properties. The inability to adapt is one unique property of pain sensation from other senses, which would be dampened by constant stimulations (12). In this sense, SCP lasts long enough to trigger significant changes in the pain pathways and potentially benefit depression recovery despite shifting homeostatic states of the body system, through fine tuning the inter-connected endocrine, immune, and nervous 
Review

systems. Similarly, exercise, fish oil consumption, controlled breathing, and other relaxation therapies increase vagus nerve activities and decreasing proinflammatory cytokine release, in agreement with observed clinic benefits of vagus nerve stimulation against depression $(40-42,53)$.

\section{Possible cellular/molecular mechanisms for the antidepressant effects of SCP}

The aforementioned transcriptomic study on several major psychiatric disorders also identified genes up-regulated specifically in MDD that are enriched for $\mathrm{G}$ protein-coupled receptors, cytokine-cytokine interactions, and hormone activity pathways, which indicate the direct involvement of inflammation and HPA axis dysregulation in depression (33). In another study that investigated the overlap between genes with robust changes in expression caused by the antidepressant Mianserin (a noradrenaline uptake inhibitor) and genes involved in depressive symptoms of an aging non-psychiatric population discovered through a genomewide association (GWAS) approach, the top candidate based on relevance to mood disorders and stress response was identified as ANK3 (54). The protein encoded by $A N K 3$, ankyrin-G, has been found as an critical regulator of synapses and strongly associated with schizophrenia, depression, anxiety disorders, and autism $(55,56)$. These suggest the direct role of neuronal signaling and synapses in depression and other psychiatric disorders $(55,56)$. Potentially SCP can function through the same pathway(s) to counteract the damaging effect of depression.

Neuron plasticity as one of their most fundamental and intrinsic properties requires neurons to change their cellular behaviors and intracellular functions upon signaling triggered by stimulations from either inside or outside the cells. Integration of cellular history with current environment determines the response of cells at certain space and time. Based on our limited literature studies, at the center of the link between SCP and depression is the hypothalamus, the neuroendocrine 'headquarters' in the body (58). First of all, the hypothalamus plays critical roles in nociceptive processing and pain modulation $(59,60)$. Secondly, the hypothalamus controls various endocrine systems through synthesizing and secreting specific hormones that modulate the secretion of pituitary hormones, which further control a wide range of physiological processes (61).

Physical stressors such as SCP convey excitatory information through ascending aminergic pathways toward the paraventricular nucleus (PVN) of the hypothalamus, which synthesizes corticotrophin-releasing factor $(\mathrm{CRH})$ to drive sympathetic and behavioral fight-or-flight response and to activate the HPA axis (62). The end product of HPA axis, glucocorticoids, can reach every organ by way of circulation and has a profound effect on the whole body and brain functions. Depression has been known to be associated with elevated levels of cortisol and dysfunctional feedback mechanisms within the HPA axis (38). 
Review

The complex actions of glucocorticoids mediated by a dual receptor systems, mineralocorticoid (MR) and glucocorticoid receptors (GR), as well their roles in mental health are comprehensively reviewed (39). Here we will just emphasize several pertinent points from that review: 1) the HPA axis and glucocorticoids coordinate both the initial fight-or-flight stress reactions and later recovery/adaptations, in concert with catecholamines (adrenal hormones) and neuropeptides; 2) the molecular signaling pathways that underlies neuroendocrine communication which integrates body and brain functions are extremely complex and dependent on time, space, and context; 3) the balance of $\mathrm{MR} / \mathrm{CR}$ function is critical and the relevant genetic variations and epigenetic modulations are implicated in stress response, HPA axis responsiveness, and depressive feelings; 4) a three-hit hypothesis was proposed that a combination of risk/plasticity genes with early adversity and later stressful life events generates a state vulnerable for mental disorders. Then it is possible that the response to SCP can have long-lasting and whole brain/body effect to counteract depression and associated inflammation symptoms as well.

There are two other important examples for the capacity of hypothalamus's control over the whole body, hypothalamic-pituitary-thyroid (HPT) axis and hypothalamic-pituitary-gonadal (HPG) axis, the latter of which, together with gonadotropin-releasing hormone $(\mathrm{GnRH})$ as mentioned later, has been extensively implicated in ageing and longevity. A lot of related studies have been focused on ageing and longevity, but behavior and mood can just be the other aspects of one integral system (63).

Mediobasal hypothalamus $(\mathrm{MBH})$ modulates immune responses and endocrine systems (58) and is the most sensitive brain region to nuclear factor $-\kappa \mathrm{B}(\mathrm{NF}-\kappa \mathrm{B})$ activation during the ageing process, which is a stress signal pathway that stimulate immune responses (61). In the glial cells of $\mathrm{MBH}, \mathrm{NF}-\kappa \mathrm{B}$ activation results in the production and secretion of the inflammatory cytokine tumor necrosis factor (TNF)- $\alpha$, which then leads to the activation of NF- $\kappa \mathrm{B}$ in $\mathrm{GnRH}$ neurons and reduced production of $\mathrm{GnRH}(61,64)$. Of note, $\mathrm{GnRH}$ travels within in the brain to promote brain-wide adult neurogenesis independently of a specific sex hormone (58).

There is a strikingly similar pattern of cross-talk between glial cells and neurons in studies of ATM (A-T mutated) and neurodegeneration in Drosophila melanogaster, where ATM mutations cause neurodegeneration by activating a specific Nuclear Factor- $\mathrm{B}(\mathrm{NF}-\kappa \mathrm{B})$ transcription factor, Relish, in glial cells, which regulates the immune deficiency (Imd) innate immune response (IIR) signaling pathway and leads to neuron and glial cells death through an yet unknown mechanism $(65,66)$.

However, how activation of HPA/HPT/HPG pathway is triggered at the molecular level has not been fully illustrated. Here, we propose that enhanced perception and neuronal activity upon exposure to sustained stressors lead to unusually high levels of DNA double strand break (DSB), which subsequently activate DNA 
Review

damage responses (DDR) in an overwhelming way that triggers inflammation in the nervous system. The long-term effect depends on the subsequent response, where efficient repair would restore the system and potentially enhance plasticity, but impaired capacity to repair (for example, ATM mutants) contributes to cell death, neurodegeneration, and/or other pathological conditions. Alternatively, the higher neuronal activity in the first place is sufficient to cause downstream effects, such neuroendocrine regulation through hypothalamus. For example, the meditation-associated SCP may sequentially trigger strong neuronal activity within certain areas of the brain (such as hypothalamus), induce DNA DSBs and DDR in the neural cells, and/or activate innate immune response (IIR) in glial cells, and ultimately modulates immune and endocrine systems through the mammalian HPA axis.

It is only in recent years that DNA DSBs and DDR have been recognized to have a much wider presence and a much more profound involvement in normal cellular functions, especially in the nervous system. For example, DDR signaling is required for efficient RNA polymerase II (Pol II) pause release and transcriptional signaling (67). Physiological neural activity, such as during learning and exploring a novel environment, has been shown to cause DNA DSBs in mice neurons (68). A more recent study found that neuronal activity stimulation triggers DNA DSBs in the promoters of some early-response genes, including Fos, Npas4, and Egr1, to induce gene expression (69). These studies as well as others not mentioned here due to limited space, suggest that activityinduced DSB formation by Topo II might be a conserved mechanism to respond to stimulations and to rapidly induce gene expression (70).

DNA strand breaks (either single strand or double strand break) activate Poly (ADP-ribose) polymerase (PARP) for DNA repair, whereas excessive activation of PARP depletes tissue stores of its substrate, nicotinamide adenine dinucleotide (NAD), and results in depletion of ATP and cell death (71). More intriguingly, PARP inhibitors provide broad protection from DNA damage-involved tissue damages in animal models of diseases as diverse as diabetes, vascular stroke, and ischaemic injury (71).

Our hypothesis on the involvement of DNA DSB and DDR agrees well with the newly emerging field of neuroepigenetics, where DNA and histone modifications are closely associated with neuronal activity-induced gene expression regulation, plasticity, and survival, whereas alterations in these neuroepigenetic mechanisms are linked to neurodevelopmental, psychiatric, and neurodegenerative disorders (70). For example, ATM helps to recruit Sirt1 to DSBs, which in return enhances ATM activity and stimulates the neuroprotective class I histone deacetylase HDAC1 (72). The collaboration between Sirt1, ATM, and HDAC1 is required for DSB repair though the nonhomologous end-joining pathway (NHEJ) to maintain genomic stability in post-mitotic neurons (73). In line with the close relationship between neuronal activity-triggered DSBs, DDR, and neuroepigenetic pathways, chromatin accessibility landscape was found to be modified $1 \mathrm{~h}$ after neuronal activation, with enrichment of gained-open regions at 
Review

active enhancers and at binding sites of AP-1 complex subunits, including Fos and FosB, both of which also have identified DNA DSBs within their respective promoters $(69,74)$.

Sirt1, as well as other members of sirtuins (the silent information regulator 2 (Sir2) family of $\mathrm{NAD}^{+}$-dependent protein deacetylases and deacylases), plays critical roles in the brain, especially in the hypothalamus, in regulating diverse functions that includes feeding behavior, endocrine modulation, physiological rhythms, and emotions (61). The hypothalamic-pituitary axis (such as HPA, HPT, and HPG), as discussed earlier, is modulated by Sirt1 through the synthesis and secretion of hormones in the hypothalamus and pituitary gland (61), whereas Sirt2 could specifically function as an inhibiter of microglia-mediated inflammation and neurotoxicity (75). Since NAD+ is required for all the enzymatic activities, sirtuins can also function as sensors of the cellular energy status (61).

In the case of diseases like A-T, defects in DDR cause neurodegeneration, whereas chronic pain leads to cortisol de-sensitization and other immune- and neuro- malfunctions. Thus, it seems that maintaining a balanced DDR is critical for cellular functions, where SCP can stimulate the system in a controlled manner to activate the functionality of immune, nervous, and endocrine modulations, which counteracts both the local neuro-inflammatory imbalance caused by SCP and also the systematically maladaptive states of depression as a beneficial byproduct.

Deep brain stimulation (DBS), which is an established treatment for movement disorders and Parkinson's disease, has also been actively explored for its effectiveness of the management of treatment-resistant depression (TRD) in preclinical studies and clinical trials. An integrated review by Dandekar et al. provides useful insights that 1) stimulation of pertinent brain regions displayed differential effects on mood transition in TRD patients, 2) stimulation parameters and neuroanatomical locations affected DBS-associated antidepressant effects, and 3) modulatory influence on monoamine neurotransmitters in target regions or interconnected brain network could be the potential mechanism (76).

In a recent study to investigate the mechanisms of DBS benefits using a mouse model of Rett syndrome (RTT), a neurodevelopmental disorder caused by mutations in the gene encoding methyl-CpG-binding protein 2 (MeCP2) (77), genes involved in synaptic function, cell survival, and neurogenesis are upregulated and about $25 \%$ of the genes differentially expressed in Mecp2-null mice are normalized upon 45 min of forniceal DBS with a 20 min recovery period (78). Interestingly, a significant portion of genes induced by DBS are involved in apoptosis regulation, which is an important part of DDR $(79,80)$, suggesting that DNA DSBs likely take place and trigger those transcriptional changes during DBS. More intriguingly, $17 \%$ of the genes downregulated in post-mortem human brain tissues from patients with MDD are found in the DBS-upregulated genes, whereas $35 \%$ of the genes upregulated by treatment with antidepressant fluoxetine, a selective serotonin reuptake inhibitor, overlap with genes upregulated by forniceal DBS (78). Compared to DBS, SCP represents a natural 
Review

option that is likely more precisely targeting and much less invasive, and thus can potentially apply to a wider population of MDD patients for better treatment outcomes.

\section{Conclusions and future directions}

Acute pain can be useful to trigger reflex to prevent injury and chronic pain is detrimental, but what lies in between? SCP may exhibit different properties from those two types of pain and represent an exciting new research area that has been largely neglected to date. Our hypothesis remains to be proved and verified in other MDD patients and the proposed potential mechanisms merit future investigation. Even though there won't be a silver bullet, the knowledge gained through future studies will further support and advance our understanding of the amazing plasticity of the brain, as well as help devise personalized lifestyle/medicine strategy to prevent and cure diseased brains. 
Review

\section{Acknowledgements and disclosures}

I am indebted to Dr. Christina M. Hull's support of me as a research associate on antifungal development and encouragement of transition from microbiology to depression-related neuroscience.

The authors report no biomedical financial interests or potential conflicts of interest. 
Review

\section{References:}

1. Kessler RC, Berglund P, Demler O, Jin R, Koretz D, Merikangas KR, et al. (2003): The Epidemiology of Major Depressive Disorder: Results From the National Comorbidity Survey Replication (NCS-R). JAMA. 289: 30953105.

2. Murray CJL, Vos T, Lozano R, Naghavi M, Flaxman AD, Michaud C, et al. (2012): Disability-adjusted life years (DALYs) for 291 diseases and injuries in 21 regions, 1990-2010: a systematic analysis for the Global Burden of Disease Study 2010. Lancet. 380: 2197-2223.

3. Insel TR, Wang PS (2009): The STAR*D Trial: Revealing the Need for Better Treatments. PS. 60: 1466-1467.

4. Rush AJ, Trivedi MH, Wisniewski SR, Nierenberg AA, Stewart JW, Warden D, et al. (2006): Acute and Longer-Term Outcomes in Depressed Outpatients Requiring One or Several Treatment Steps: A STAR ${ }^{\star}$ Report. AJP. 163: $1905-1917$.

5. Yohn CN, Gergues MM, Samuels BA (2017): The role of 5-HT receptors in depression. Molecular Brain. 10: 28.

6. Kasala ER, Bodduluru LN, Maneti Y, Thipparaboina R (2014): Effect of meditation on neurophysiological changes in stress mediated depression. Complement Ther Clin Pract. 20: 74-80.

7. Simkin DR, Black NB (2014): Meditation and mindfulness in clinical practice. Child Adolesc Psychiatr Clin N Am. 23: 487-534. 
Review

8. Hirsch JK, Sirois FM, Molnar D, Chang EC (2016): Pain and Depressive Symptoms in Primary Care: Moderating Role of Positive and Negative Affect. The Clinical Journal of Pain. 32: 562.

9. Geisser ME, Roth RS, Theisen ME, Robinson ME, Riley JLI (2000): Negative Affect, Self-Report of Depressive Symptoms, and Clinical Depression: Relation to the Experience of Chronic Pain. The Clinical Journal of Pain. 16: 110 .

10. Okifuji A, Turk DC (2016): Chapter 9 - Chronic Pain and Depression: Vulnerability and Resilience. In: al'Absi M, Flaten MA, editors. Neuroscience of Pain, Stress, and Emotion. San Diego: Academic Press, pp 181-201.

11. Zhu Z, Galatzer-Levy IR, Bonanno GA (2014): Heterogeneous depression responses to chronic pain onset among middle-aged adults: a prospective study. Psychiatry Res. 217: 60-66.

12. Cervero F (2012): Understanding Pain: Exploring the Perception of Pain. MIT Press.

13. Fein A (2012): Nociceptors and the perception of pain. University of Connecticut Health Center. 4: 61-67.

14. Lunn MP, Hughes RA, Wiffen PJ (2014): Duloxetine for treating painful neuropathy, chronic pain or fibromyalgia. The Cochrane Library. John Wiley \& Sons, Ltd. doi: 10.1002/14651858.CD007115.pub3.

15. Tylš F, Páleníček T, Horáček J (2014): Psilocybin--summary of knowledge and new perspectives. Eur Neuropsychopharmacol. 24: 342-356. 
Review

16. Nichols DE (2016): Psychedelics. Pharmacol Rev. 68: 264-355.

17. Sleigh J, Harvey M, Voss L, Denny B (2014): Ketamine - More mechanisms of action than just NMDA blockade. Trends in Anaesthesia and Critical Care. 4: 76-81.

18. Zarate CA, Brutsche NE, Ibrahim L, Franco-Chaves J, Diazgranados N, Cravchik A, et al. (2012): Replication of ketamine's antidepressant efficacy in bipolar depression: a randomized controlled add-on trial. Biol Psychiatry. 71: 939-946.

19. Lapidus KAB, Levitch CF, Perez AM, Brallier JW, Parides MK, Soleimani L, et al. (2014): A randomized controlled trial of intranasal ketamine in major depressive disorder. Biol Psychiatry. 76: 970-976.

20. DiazGranados N, Ibrahim LA, Brutsche NE, Ameli R, Henter ID, Luckenbaugh DA, et al. (2010): Rapid Resolution of Suicidal Ideation After a Single Infusion of an \&lt;span class='bold-italic'\&gt;N\&lt;/span\&gt;Methyl-\&lt;span class='smallcaps'\&gt;d\&lt;/span\&gt;-Aspartate Antagonist in Patients With Treatment-Resistant Major Depressive Disorder. J Clin Psychiatry. 71: 1605-1611.

21. Price RB, losifescu DV, Murrough JW, Chang LC, Al Jurdi RK, lqbal SZ, et al. (2014): Effects of ketamine on explicit and implicit suicidal cognition: a randomized controlled trial in treatment-resistant depression. Depress Anxiety. 31: 335-343.

22. Zarate CA, Singh JB, Carlson PJ, Brutsche NE, Ameli R, Luckenbaugh DA, et al. (2006): A Randomized Trial of an N-methyl-D-aspartate Antagonist 
Review

in Treatment-Resistant Major Depression. Arch Gen Psychiatry. 63: 856864.

23. Berman RM, Cappiello A, Anand A, Oren DA, Heninger GR, Charney DS, Krystal JH (2000): Antidepressant effects of ketamine in depressed patients. Biological Psychiatry. 47: 351-354.

24. Murrough JW, losifescu DV, Chang LC, Al Jurdi RK, Green CE, Perez AM, et al. (2013): Antidepressant Efficacy of Ketamine in Treatment-Resistant Major Depression: A Two-Site Randomized Controlled Trial. AJP. 170: $1134-1142$.

25. Diazgranados N, Ibrahim L, Brutsche NE, Newberg A, Kronstein P, Khalife S, et al. (2010): A Randomized Add-on Trial of an N-methyl-D-aspartate Antagonist in Treatment-Resistant Bipolar Depression. Arch Gen Psychiatry. 67: 793-802.

26. Singh JB, Fedgchin M, Daly EJ, De Boer P, Cooper K, Lim P, et al. (2016): A Double-Blind, Randomized, Placebo-Controlled, Dose-Frequency Study of Intravenous Ketamine in Patients With Treatment-Resistant Depression. AJP. appi.ajp.2016.16010037.

27. Singh JB, Fedgchin M, Daly E, Xi L, Melman C, De Bruecker G, et al. (2016): Intravenous Esketamine in Adult Treatment-Resistant Depression: A Double-Blind, Double-Randomization, Placebo-Controlled Study. Biol Psychiatry. 80: 424-431.

28. Zanos P, Gould TD (2018): Mechanisms of ketamine action as an antidepressant. Molecular Psychiatry. 23: 801-811. 
Review

29. Loix S, De Kock M, Henin P (2011): The anti-inflammatory effects of ketamine: state of the art. Acta Anaesthesiol Belg. 62: 47-58.

30. Najjar S, Pearlman DM, Alper K, Najjar A, Devinsky O (2013):

Neuroinflammation and psychiatric illness. Journal of Neuroinflammation. 10: 816 .

31. Liu Y, Ho RC-M, Mak A (2012): Interleukin (IL)-6, tumour necrosis factor alpha (TNF- $\alpha$ ) and soluble interleukin-2 receptors (sIL-2R) are elevated in patients with major depressive disorder: a meta-analysis and metaregression. J Affect Disord. 139: 230-239.

32. Vogelzangs N, Duivis HE, Beekman ATF, Kluft C, Neuteboom J, Hoogendijk W, et al. (2012): Association of depressive disorders, depression characteristics and antidepressant medication with inflammation. Trans/ Psychiatry. 2: e79.

33. Gandal MJ, Haney JR, Parikshak NN, Leppa V, Ramaswami G, Hartl C, et al. (2018): Shared molecular neuropathology across major psychiatric disorders parallels polygenic overlap. Science. 359: 693-697.

34. Müller N, Schwarz MJ (2007): The immune-mediated alteration of serotonin and glutamate: towards an integrated view of depression. Molecular Psychiatry. 12: 988-1000.

35. Pfau ML, Ménard C, Russo SJ (2018): Inflammatory Mediators in Mood Disorders: Therapeutic Opportunities. Annual Review of Pharmacology and Toxicology. 58: 411-428. 
Review

36. Hodes GE, Kana V, Menard C, Merad M, Russo SJ (2015): Neuroimmune mechanisms of depression. Nature Neuroscience. 18: 1386-1393.

37. Jeon SW, Kim YK (2016): Neuroinflammation and cytokine abnormality in major depression: Cause or consequence in that illness? World Journal of Psychiatry. 6: 283-293.

38. Murison R (2016): Chapter 2 - The Neurobiology of Stress. In: al'Absi M, Flaten MA, editors. Neuroscience of Pain, Stress, and Emotion. San Diego: Academic Press, pp 29-49.

39. (Ron) de Kloet ER, Joëls M (2013): Stress Research: Past, Present, and Future. In: Pfaff DW, editor. Neuroscience in the 21st Century: From Basic to Clinical. New York, NY: Springer New York, pp 1979-2007.

40. Tracey KJ (2009): Reflex control of immunity. Nature Reviews Immunology. 9: 418-428.

41. Breit S, Kupferberg A, Rogler G, Hasler G (2018): Vagus Nerve as Modulator of the Brain-Gut Axis in Psychiatric and Inflammatory Disorders. Front Psychiatry. 9. doi: 10.3389/fpsyt.2018.00044.

42. Kong J, Fang J, Park J, Li S, Rong P (2018): Treating Depression with Transcutaneous Auricular Vagus Nerve Stimulation: State of the Art and Future Perspectives. Front Psychiatry. 9. doi: 10.3389/fpsyt.2018.00020.

43. Wieser MJ, Pauli P (2016): Chapter 1 - Neuroscience of Pain and Emotion. In: al'Absi M, Flaten MA, editors. Neuroscience of Pain, Stress, and Emotion. San Diego: Academic Press, pp 3-27. 
Review

44. Apkarian AV, Bushnell MC, Treede R-D, Zubieta J-K (2005): Human brain mechanisms of pain perception and regulation in health and disease. Eur J Pain. 9: 463-484.

45. Price DD (2000): Psychological and Neural Mechanisms of the Affective Dimension of Pain. Science. 288: 1769-1772.

46. Stiles TC, Hrozanova M (2016): Chapter 12 - Chronic Pain and Fatigue. In: al'Absi M, Flaten MA, editors. Neuroscience of Pain, Stress, and Emotion. San Diego: Academic Press, pp 253-282.

47. Drevets WC (2001): Neuroimaging and neuropathological studies of depression: implications for the cognitive-emotional features of mood disorders. Curr Opin Neurobiol. 11: 240-249.

48. Richard J. Davidson, Diego Pizzagalli, Jack B. Nitschke, Putnam and K (2002): Depression: Perspectives from Affective Neuroscience. Annual Review of Psychology. 53: 545-574.

49. Goleman D, Davidson R (n.d.): Altered Traits by Daniel Goleman, Richard J. Davidson.

50. Zunszain PA, Anacker C, Cattaneo A, Carvalho LA, Pariante CM (2011): Glucocorticoids, cytokines and brain abnormalities in depression. Prog Neuropsychopharmacol Biol Psychiatry. 35: 722-729.

51. Duman RS, Aghajanian GK (2012): Synaptic Dysfunction in Depression: Potential Therapeutic Targets. Science. 338: 68-72. 
Review

52. Bryngelson JD, Onuchic JN, Socci ND, Wolynes PG (1995): Funnels, pathways, and the energy landscape of protein folding: a synthesis. Proteins. 21: 167-195.

53. Howland RH (2014): Vagus Nerve Stimulation. Curr Behav Neurosci Rep. 1: 64-73.

54. Rangaraju S, Levey DF, Nho K, Jain N, Andrews KD, Le-Niculescu H, et al. (2016): Mood, stress and longevity: convergence on ANK3. Molecular Psychiatry. 21: 1037-1049.

55. Smith KR, Kopeikina KJ, Fawcett-Patel JM, Leaderbrand K, Gao R, Schürmann B, et al. (2014): Psychiatric risk factor ANK3/ankyrin-G nanodomains regulate the structure and function of glutamatergic synapses. Neuron. 84: 399-415.

56. Tseng WC, Jenkins PM, Tanaka M, Mooney R, Bennett V (2015): Giant ankyrin-G stabilizes somatodendritic GABAergic synapses through opposing endocytosis of GABAA receptors. PNAS. 112: 1214-1219.

57. Duman RS, Aghajanian GK, Sanacora G, Krystal JH (2016): Synaptic plasticity and depression: new insights from stress and rapid-acting antidepressants. Nature Medicine. 22: 238-249.

58. Zhang G, Li J, Purkayastha S, Tang Y, Zhang H, Yin Y, et al. (2013): Hypothalamic programming of systemic ageing involving IKK- $\beta$, NF-KB and GnRH. Nature. 497: 211-216. 
Review

59. Dafny N, Dong WQ, Prieto-Gomez C, Reyes-Vazquez C, Stanford J, Qiao JT (1996): Lateral hypothalamus: site involved in pain modulation. Neuroscience. 70: 449-460.

60. Matharu MS (2007): The Hypothalamus, Pain, and Primary Headaches. Headache: The Journal of Head and Face Pain. 47: 963-968.

61. Satoh A, Imai S (2014): Systemic regulation of mammalian ageing and longevity by brain sirtuins. Nature Communications. 5: 4211 .

62. Kloet ER de, Joëls M, Holsboer F (2005): Stress and the brain: from adaptation to disease. Nature Reviews Neuroscience. 6: 463-475.

63. Zhang B, Gong J, Zhang W, Xiao R, Liu J, Xu XZS (2018): Brain-gut communications via distinct neuroendocrine signals bidirectionally regulate longevity in C. elegans. Genes Dev. 32: 258-270.

64. Saijo K, Winner B, Carson CT, Collier JG, Boyer L, Rosenfeld MG, et al. (2009): A Nurr1/CoREST pathway in microglia and astrocytes protects dopaminergic neurons from inflammation-induced death. Cell. 137: 47-59.

65. Petersen AJ, Rimkus SA, Wassarman DA (2012): ATM kinase inhibition in glial cells activates the innate immune response and causes neurodegeneration in Drosophila. PNAS. 109: E656-E664.

66. Petersen AJ, Katzenberger RJ, Wassarman DA (2013): The Innate Immune Response Transcription Factor Relish Is Necessary for Neurodegeneration in a Drosophila Model of Ataxia-Telangiectasia. Genetics. 194: 133-142. 
Review

67. Bunch H, Lawney BP, Lin Y-F, Asaithamby A, Murshid A, Wang YE, et al. (2015): Transcriptional elongation requires DNA break-induced signalling. Nature Communications. 6: 10191.

68. Suberbielle E, Sanchez PE, Kravitz AV, Wang X, Ho K, Eilertson K, et al. (2013): Physiologic brain activity causes DNA double-strand breaks in neurons, with exacerbation by amyloid- $\beta$. Nature Neuroscience. 16: 613621.

69. Madabhushi R, Gao F, Pfenning AR, Pan L, Yamakawa S, Seo J, et al. (2015): Activity-Induced DNA Breaks Govern the Expression of Neuronal Early-Response Genes. Cell. 161: 1592-1605.

70. Cholewa-Waclaw J, Bird A, Schimmelmann M von, Schaefer A, Yu H, Song $\mathrm{H}$, et al. (2016): The Role of Epigenetic Mechanisms in the Regulation of Gene Expression in the Nervous System. J Neurosci. 36: 11427-11434.

71. Pieper AA, Verma A, Zhang J, Snyder SH (1999): Poly (ADP-ribose) polymerase, nitric oxide and cell death. Trends Pharmacol Sci. 20: 171181.

72. Dobbin MM, Madabhushi R, Pan L, Chen Y, Kim D, Gao J, et al. (2013): SIRT1 collaborates with ATM and HDAC1 to maintain genomic stability in neurons. Nature Neuroscience. 16: 1008-1015.

73. Madabhushi R, Pan L, Tsai L-H (2014): DNA damage and its links to neurodegeneration. Neuron. 83: 266-282. 
Review

74. Su Y, Shin J, Zhong C, Wang S, Roychowdhury P, Lim J, et al. (2017):

Neuronal activity modifies the chromatin accessibility landscape in the adult brain. Nature Neuroscience. 20: 476-483.

75. Pais TF, Szegő ÉM, Marques O, Miller-Fleming L, Antas P, Guerreiro P, et al. (2013): The NAD-dependent deacetylase sirtuin 2 is a suppressor of microglial activation and brain inflammation. The EMBO Journal. 32: 2603-2616.

76. Dandekar MP, Fenoy AJ, Carvalho AF, Soares JC, Quevedo J (2018): Deep brain stimulation for treatment-resistant depression: an integrative review of preclinical and clinical findings and translational implications. Molecular Psychiatry. 23: 1094-1112.

77. Ip JPK, Mellios N, Sur M (2018): Rett syndrome: insights into genetic, molecular and circuit mechanisms. Nature Reviews Neuroscience. 19: 368-382.

78. Pohodich AE, Yalamanchili H, Raman AT, Wan Y-W, Gundry M, Hao S, et al. (2018): Forniceal deep brain stimulation induces gene expression and splicing changes that promote neurogenesis and plasticity. eLife Sciences. 7: e34031.

79. Borges HL, Linden R, Wang JY (2008): DNA damage-induced cell death: lessons from the central nervous system. Cell Research. 18: 17-26.

80. Rich T, Allen RL, Wyllie AH (2000, October 12): Defying death after DNA damage. Nature. Special Features. 